\begin{tabular}{|c|l|}
\hline Title & Decreased IL-10 production mediated by Toll-like receptor 9 in B cells in multiple sclerosis \\
\hline Author(s) & $\begin{array}{l}\text { Hirotani, Makoto; Niino, Masaaki; Fukazawa, Toshiyuki; Kikuchi, Seiji; Y abe, Ichiro; Hamada, Shinsuke; Tajima, } \\
\text { Y asutaka; Sasaki, Hidenao }\end{array}$ \\
\hline Citation & $\begin{array}{l}\text { Journal of Neuroimmunology, 221(1-2), 95-100 } \\
\text { https:/doi.org/10.1016/.jneuroim.2010.02.012 }\end{array}$ \\
\hline Issue Date & 2010-04-15 \\
\hline Doc URL & http://hdl.handle.net/2115/43147 \\
\hline Type & article (author version) \\
\hline File Information & JN221-1-2_95-100.pdf \\
\hline
\end{tabular}

Instructions for use 


\section{Decreased IL-10 production mediated by Toll-like receptor 9 in B cells in multiple sclerosis}

Makoto Hirotani $^{1}$, Masaaki Niino ${ }^{1}$, Toshiyuki Fukazawa ${ }^{2}$, Seiji Kikuchi ${ }^{3}$, Ichiro Yabe ${ }^{1}$, Shinsuke Hamada $^{4}$, Yasutaka Tajima ${ }^{5}$ and Hidenao Sasaki ${ }^{1}$

${ }^{1}$ Department of Neurology, Hokkaido University Graduate School of Medicine, Sapporo, Japan

${ }^{2}$ Sapporo Neurology Clinic, Sapporo, Japan

${ }^{3}$ Department of Neurology, Sapporo Minami National Hospital, Sapporo, Japan

${ }^{4}$ Hokuyukai Neurological Hospital, Sapporo, Japan

${ }^{5}$ Department of Neurology, Sapporo City General Hospital, Sapporo, Japan

Corresponding author: Masaaki Niino, Department of Neurology, Hokkaido University

Graduate School of Medicine, Kita-15, Nishi-7, Kita-ku, Sapporo 060-8638 Japan.

E-mail: niino@med.hokudai.ac.jp

Tel: +81-11-706-6028

FAX: +81-11-700-5356

Running Title: TLR9 and B cells in MS 


\begin{abstract}
The complexity of the roles of Toll-like receptors (TLRs) is attributable to their ability to promote or suppress autoimmune diseases. Recent studies have demonstrated that B cells regulate autoimmune diseases, including multiple sclerosis (MS), by producing interleukin (IL)-10. By using CpG DNA as a TLR9 agonist, we investigated the immunoregulatory functions of B cell via TLR9 in MS. Our results indicate that TLR9-mediated IL-10 production by B cells was significantly decreased in MS, and this decrease is likely due to decreased TLR9 expression in memory B cells, suggesting a role of TLR9 in immunoregulation in MS.
\end{abstract}

Key words: MS, TLR9, CpG DNA, immunoregulation 


\section{Introduction}

Multiple sclerosis (MS) is an inflammatory, demyelinating disease that affects the central nervous system (CNS). Autoimmunity plays a major role in susceptibility to and development of MS. It has been demonstrated that bacterial or viral infections can trigger MS onset and/or relapse via molecular mimicry (Zhang et al., 2008) and induction of Toll-like receptor (TLR) signaling (Iwasaki and Medzhitov, 2004). The innate immune response, which is the first- line of defense against microbial pathogens, was recently found to participate via TLR signaling in the regulation of the antigen-specific adaptive immune response during MS pathogenesis (Boonstra et al., 2006). Studying the direct immunoregulatory functions of TLR-signaling pathways will potentially establish the validity of the long-held notion that bacterial and viral infections protect against the subsequent development of autoimmune diseases (Lehmann and Ben-Nun, 2002). This "hygiene hypothesis” offers an explanation for the high prevalence of autoimmune diseases, including MS (Bach, 2002).

TLRs are a class of germline-encoded receptors that can be activated by pathogen-associated molecular patterns (PAMPs). These receptors are essential for the generation of adaptive immune responses against a wide variety of microbial and nonmicrobial components (Iwasaki and Medzhitov, 2004; Takeda et al., 2003). In humans, 10 TLRs and their corresponding agonists have been described. TLR9 was identified in B cells and plasmacytoid dendritic cells (DCs), and unmethylated CpG DNA was identified as a TLR9 agonist.

The complexity of TLR roles in the pathogenesis of autoimmune diseases is attributable to their ability to promote or suppress autoimmune diseases. Prinz and colleagues demonstrated that mice deficient in MyD88, an adaptor protein for all TLRs except TLR3, are resistant to experimental autoimmune encephalomyelitis (EAE) induced by a peptide derived from myelin oligodendrocyte glycoprotein (MOG), and that TLR9 mediates pathogenesis in this model (Prinz et al., 2006). On the other hand, Marta and colleagues demonstrated that TLR4- and 
TLR9-deficient mice exhibit more severe EAE symptoms than those observed in wild-type mice, and that TLR4 and TLR9 have regulatory roles in EAE (Marta et al., 2008).

B cells express TLR9 intracellularly, produce antibodies, and contribute to pathogenesis during acquired immune responses by producing autoantibodies, leading to autoimmunity. B cells also contribute to immune responses by functioning as antigen- presenting cells and by secreting cytokines. In addition to these potentially pathogenic roles, experimental evidence indicates that regulatory B cells develop in different murine autoimmunity models and suppress the progression of immune-mediated diseases. Detailed studies of the underlying mechanisms revealed that B cells regulate EAE by producing IL-10 (Fillatreau et al., 2002), while B cells from MS patients produce low levels of IL-10 (Duddy et al., 2007). It is conceivable that multiple activation pathways, including those mediated by B-cell receptor (BCR), CD40, and TLR, regulate various functions of B cells (Vos et al., 2000).

Considering this background, we aimed to elucidate the mechanisms underlying the immunoregulatory functions of TLR9 in MS by studying B-cell cytokine profiles and their responses upon stimulation with CpG DNA. We demonstrated that TLR9-mediated IL-10 production in B cells is decreased in MS, and this decrease is likely due to decreased TLR9 expression in memory B cells. 


\section{Materials and methods}

\subsection{MS patients and healthy individuals}

We recruited 36 patients with relapsing-remitting MS [age, $36.2 \pm 10.5$ years (mean \pm SD)] and 10 age- and sex-matched healthy subjects (age, $34.8 \pm 11.0$ years) (Table 1). MS diagnosis was established according to Poser's criteria (Poser et al., 1983), and patients with optico-spinal MS or relapsing Devic's disease were excluded. In our study, 6 patients were in the relapsing phase (age, $32.3 \pm 14.8$ years) and 30 in the remitting phase (age, $37.0 \pm 9.6$ years). Of the patients in the remitting phase, 10 (age, $39.6 \pm 4.4$ years) were treated with IFN $\beta$-1a (30 $\mu$ g by intramuscular injection once a week); 10 (age, $35.5 \pm 9.0$ years) were treated with IFN $\beta-1 b$ (8 million units by subcutaneous injection every alternate day); and the remaining 10 (age, $37.7 \pm$ 12.7 years) did not receive any disease-modifying therapy (DMT). The abovementioned patient groups were consistently treated with IFN $\beta$-1a and IFN $\beta$-1b for at least 6 months. The study was approved by the ethical committee of Hokkaido University Hospital. All subjects granted written consent forms.

\subsection{Isolation of B cells}

Venous blood was drawn into tubes containing ethylene- diamine- tetraacetic acid (EDTA) and peripheral blood mononuclear cells (PBMCs) were freshly separated by standard density centrifugation and Ficoll-Paque (Pharmacia Biotech). CD19-positive B cells were purified using magnetic cell sorting (MACS; Miltenyi Biotec) by negative immunomagnetic selection according to the manufacturer's protocol without any modification. Isolated B cells had a purity of $>93 \%$ as assessed by flow cytometry.

\subsection{Flow cytometry}


To perform cell surface staining, we added PE-conjugated anti-human CD5, CD27, CD80, and CD86 antibodies (BD Biosciences, San Jose, CA, USA), and PE-Cy5-conjugated anti-human CD19 (BD Biosciences) to whole blood, which was incubated for $30 \mathrm{~min}$ on ice. For intracellular staining, we used Intraprep ${ }^{\mathrm{TM}}$ (Beckman Coulter, Miami, FL, USA) for fixation and permeabilization. For intracellular staining, the cells were incubated with $5 \mu$ l FITC-conjugated anti-human TLR9 (Imgenex, San Diego, CA, USA) for 30 min according to the manufacturer's protocol without any modification. The staining was conducted in PBS supplemented with 2\% fatal calf serum (FCS). Cells were analyzed immediately on a FACSCalibur flow cytometer (BD Biosciences) with CellQuest software (BD Biosciences).

\subsection{B cell stimulation and cytokine responses}

Isolated B cells were cultured at a density of $2 \times 10^{5}$ cells per well in 24-well, flat-bottom plates in complete RPMI 1640 medium supplemented with 10\% FCS (HyClone Laboratories, Logan, UT). CpG oligodeoxynucleotide 2006 (5'-TCGTCGTTTTGTCGTTTTGTCGTT-3') was used optimally at $0.25 \mu \mathrm{M}$. The CpG-stimulated cells were then cultured for 24 hours at $37^{\circ} \mathrm{C}$ in a humidified atmosphere containing $5 \% \mathrm{CO}_{2}$ before the supernatants were collected. Aliquots of the supernatants were prepared and frozen at $-80{ }^{\circ} \mathrm{C}$ until batch analysis was performed for measuring cytokines by standard ELISA. IL-10, IL-12 (p40), and tumor necrosis factor- $\alpha$ (TNF- $\alpha$ ) were assayed using OptEIA ELISA kits (BD Biosciences) according to the manufacturer's protocol. Lymphotoxin- $\alpha$ (LT- $\alpha$ ) was assayed using ELISA plates coated overnight with $2 \mu \mathrm{g} / \mathrm{ml}$ anti-human LT- $\alpha$ (BD Biosciences). After the plates were washed and blocked with $10 \%$ FCS, the samples were incubated for 2 hours at room temperature. The plates were washed once again and $1 \mu \mathrm{g} / \mathrm{ml}$ biotinylated anti-human LT- $\alpha$ (BD Biosciences) with avidin-conjugated HRP were added for 1 hour at room temperature. After further washing, color was developed using tetramethylbenzidine for the OptEIA kits. 


\subsection{Statistical analysis}

Results were expressed as means \pm standard deviation (SD) and considered statistically significant when $p<0.05$. Analysis of variance (ANOVA) was used to compare cytokine production and antigen expression in B cells among all the patient groups, and Tukey-Kramer comparison tests were subsequently performed. Spearman rank correlation was used for correlation analysis. 


\section{Results}

3.1. Abnormal B cell-cytokine network in MS

The average levels of IL-10 derived from unstimulated B cells did not significantly differ among patients with relapsing MS, MS patients without DMT, MS patients with IFN $\beta-1 \mathrm{a}$ treatment, MS patients with IFN $\beta$-1b treatment, and healthy individuals. The mean levels of IL-10 produced by B cells stimulated with CpG DNA minus IL-10 levels produced by unstimulated B cells ( $\Delta \mathrm{IL}-10 \mathrm{in} \mathrm{pg} / \mathrm{ml})$ for the abovementioned groups were as follows: patients with relapsing MS, $2.69 \pm 5.84$; MS patients without DMT, decreased to $0.17 \pm 4.00$; MS patients treated with IFN $\beta-1 \mathrm{a}, 0.27 \pm 6.0$; MS patients treated with IFN $\beta-1 \mathrm{~b}, 2.41 \pm 3.17$; and healthy subjects, $13.13 \pm 14.59 \mathrm{pg} / \mathrm{ml}$ (Figure $1 \mathrm{~A}$ ). These results show that IL-10 production via TLR9 in B cells was significantly decreased in MS patients $(p<0.001)$. Neither the disease phase (remitting or relapsing) nor DMT affected IL-10 production via TLR9 in B cells.

The average levels of IL-12 derived from unstimulated B cells did not significantly differ among the abovementioned groups. The mean levels of IL-12 (p40) produced by B cells stimulated with CpG DNA minus the IL-12 levels produced by unstimulated B cells ( $\Delta \mathrm{IL}-12)$ were $31.31 \pm 29.74,0.99 \pm 14.15$, and $0.53 \pm 1.07 \mathrm{pg} / \mathrm{ml}$ in patients with relapsing MS, MS patients without DMT, and healthy subjects, respectively (Figure 1B). Furthermore, $\Delta \mathrm{IL}-12$ was decreased to $6.22 \pm 29.03$ in MS patients treated with IFN $\beta-1$ a and to $1.35 \pm 33.62 \mathrm{pg} / \mathrm{ml}$ in those treated with IFN $\beta$-1b. These results show that IL-12 (p40) production via TLR9 in B cells was significantly greater in patients with relapsing MS than in all the other groups $(p<0.05)$.

The mean levels of $\Delta \mathrm{TNF}-\alpha$ in $\mathrm{pg} / \mathrm{ml}$ for the abovementioned groups were as follows: patients with relapsing MS, $7.33 \pm 11.23$; MS without DMT, $5.50 \pm 10.03$; MS patients with IFN $\beta-1 \mathrm{a}$ treatment, $5.34 \pm 15.0$; MS patients with IFN $\beta$-1b treatment, $1.88 \pm 13.06$; and healthy subjects, $2.56 \pm 6.29$ (Figure 1C). No significant differences were found in TNF- $\alpha$ production via TLR9 
in B cells among the above groups.

The mean amount of $\Delta \mathrm{LT}-\alpha$ were under zero in all the abovementioned groups (data not shown).

3.2. Decreased mean fluorescence intensity (MFI) of TLR9 in memory B cells in MS

To investigate the profiles of B cells, we assessed the expression of the surface antigens CD5, CD27, CD80, and CD86 and measured intracellular TLR9 in CD19-positive B cells. The proportion of CD27-positive B cells (memory B cells) was significantly higher in patients with relapsing MS than in MS patients treated with IFN $\beta-1$ a or IFN $\beta-1 b(p<0.05)$ (Figure 2A, 2B). Furthermore, this proportion did not differ significantly among the other groups. The proportion of CD80-positive B cells was also significantly higher in patients with relapsing MS than in the other study groups (Figure 2A, 2C). Moreover, the proportion of CD86-positive B cells was significantly lower in MS patients treated with IFN $\beta$-1b than in those who did not receive DMT (Figure 2A, 2D). There were no significant differences in proportion of CD5-positive B cells among all the other groups (data not shown).

The MFI of TLR9 in B cells did not differ significantly among all the groups (Figure 3A); however, the average TLR9 MFI in memory B cells was significantly lower in the MS patients than in healthy individuals ( $p<0.01$; Figure 3B, 3C).

3.3. Correlation between TLR9-mediated IL-10 production in B cells and TLR9 MFI in memory B cells

To evaluate the relationship between cytokine production via CpG DNA and TLR9 expression in memory B cells, we examined the correlation between TLR9 MFI in memory B cells and IL-10 or IL-12 (p40) production in CpG-DNA-stimulated B cells. A significantly positive correlation was found between B cell-mediated IL-10 production and TLR9 MFI in B cells from 
all the subjects ( $p<0.001, r=0.752$ ) (Figure 4 ), but no correlation was found between IL-12 (p40) production and TLR9 MFI in memory B cells (data not shown). 


\section{Discussion}

The major findings of the present study are as follows: (1) IL-10 production by CpG-DNA-stimulated B cells was significantly lower in MS patients than in healthy, control individuals, (2) IL-12 production by B cells was significantly higher in patients with relapsing MS than in those with remitting MS or healthy individuals, (3) the TLR9 MFI in memory B cells was significantly lower in MS patients than in the controls, and (4) a significant, positive correlation was found between TLR9 MFI in memory B cells and IL-10 production. These results suggest that TLR9-mediated cytokine production by B cells could be dysregulated in MS patients and that the decrease in TLR9-mediated IL-10 production by B cells may partly be associated with low TLR9 expression in memory B cells.

TLRs play complex roles in the pathogenesis of autoimmunity because they can either promote or suppress autoimmune diseases. Lampropoulou and colleagues demonstrated that MyD88, an adaptor protein for all TLRs except for TLR3, plays a dual role in EAE because it controls both disease initiation and termination (Lampropoulou et al., 2008). Moreover, Prinz

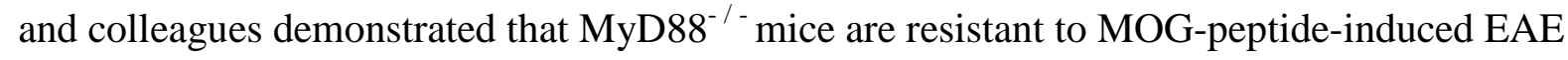
and that TLR9 modulates pathogenesis in this model (Prinz et al., 2006). In contrast, Marta and colleagues demonstrated that TLR9-deficient mice exhibit more severe EAE symptoms than wild-type mice owing to increased levels of IL-6 (Marta et al., 2008). They also reported that the critical difference between disease- promotion and disease- suppression in TLR9-deficient mice with EAE depends on the immunization regimen used for EAE induction (Marta et al., 2008). For examples, Marta and colleagues used MOG for immunization; MOG can adequately prime encephalitogenic Th17 cells. In contrast, Prinz and colleagues used an MOG-derived peptide in an immunization regimen that required a booster to induce EAE. The difference in immunization may explain the differences in the results of the 2 studies, because unlike immunization with MOG peptide, immunization with MOG protein induces pathogenic B cell responses and 
demyelinating antibodies that are similar to those in human MS. Mechanisms of TLR9 regulation have not been fully understood because of its dual actions on both B cells and other cells such as dendritic cells (DCs). However, the data collected by Marta and colleagues strongly suggest that B cell responses, including immunoregulation, in their model are more physiologically relevant than those in the model used by Prinz and colleagues. Our results show that the TLR9-mediated immunoregulatory functions of B cells are dysregulated in MS.

B cell-derived IL-10 is considered to be insufficient for regulating autoimmune diseases. The evidence for the regulatory functions of B cells in autoimmune diseases was first provided by Wolf and colleagues who demonstrated that B10.PL mice lacking B cells suffer from an unusually severe and chronic form of EAE (Wolf et al., 1996). The B cell-mediated regulation of EAE is associated with suppression of Th17 cells; IL-10 from TLR-activated B cells inhibits secretion of Th17 and promotes secretion of IL-6, IL-12, transforming growth factor- $\beta$ (TGF- $\beta$ ), and IL-23 (McGeachy et al., 2007). These findings suggest that B cell-derived IL-10 plays an important role in suppressing T cell-mediated autoimmunity in EAE. Duddy and colleagues identified IL-10-producing B cells in MS patients and demonstrated that B cells from these patients produce less amount of IL-10, suggesting that these B cells have regulatory roles in MS (Duddy et al., 2007). In that study, B cells were stimulated through CD40 alone or through dual BCR and CD40 activation.

All B cell subsets produce IL-10 in response to TLR9 activation; however, the immune regulatory functions of B cell subpopulations may significantly differ. Indeed, Bernasconi and colleagues have demonstrated that expression of TLR9 differs in naïve and memory B cells and that CpG DNA selectively activates memory B cells (Bernasconi et al., 2003). TLR9 is expressed at low levels in human naïve B cells; it is rapidly up-regulated by BCR and expressed at high constitutive levels in memory B cells. Consistent with these observations, it was reported that CpG DNA directly acts on memory B cells and induces their proliferation and 
differentiation into Ig-secreting cells. In contrast, naïve B cells require synergistic stimulation by anti-Ig and CpG to exhibit the same results (Bernasconi et al., 2003). Our data demonstrated a positive correlation between B cell IL-10 production and TLR9 expression in memory B cells, suggesting that CpG DNA may activate memory B cells selectively and induce IL-10 production.

IFN $\beta-1 \mathrm{a}$ and IFN $\beta-1 \mathrm{~b}$ have been used for many years as a first- line of therapy for relapsing-remitting MS. Their efficacy in suppressing disease activity has been well documented in large, randomized, placebo-controlled clinical trials (PRISMS Study Group, 1998; IFN- $\beta$ Multiple Sclerosis Study Group, 1993). IFN $\beta$ is known to be secreted during innate immune responses to viral infections, but the physiological role of endogenous type-I IFN in the regulation of the adaptive immune response is poorly understood. Zhang and colleagues have proposed that treatment with IFN $\beta$ - $1 \mathrm{a}$ induces TLR7 expression; inhibits IL-1 $\beta$, TGF- $\beta$, and IL-23, but induces IL-27 secretion by monocyte-derived DC. These cytokines collectively inhibit DC-mediated Th17 cell differentiation (Zhang et al., 2009). In our study, IFN $\beta$-1a and IFN $\beta-1 b$ affected neither IL-10 and IL-12 production nor TLR9 expression in B cells. Further studies are required to characterize the mechanisms of IFN $\beta$-induced TLR9 expression in other cells such as DC.

To our knowledge, this is the first study to report that TLR9-mediated IL-10 production in B cells is significantly decreased in MS. In addition, we have showed that the decreased IL-10 production was significantly correlated with TLR9 expression in memory B cells. Together with the results of previous studies, the present findings suggest that TLR9 in B cells plays an important role in innate and adaptive immunity and therefore in the regulation of MS pathogenesis. Furthermore, TLR9 may be a potential therapeutic target in the treatment of MS. 


\section{Acknowledgments}

This work was supported in part by a Grant-in-Aid for Scientific Research from The Ministry of Education, Culture, Sports, Science and Technology of Japan.

\section{Conflicts of interest}

The authors declare that they have no financial conflict of interest. 


\section{References}

Bach, J.F., 2002. The effect of infections on susceptibility to autoimmune encephalomyelitis. N. Engl. J. Med. 347, 911- 920.

Bernasconi, N.L., Onai, N., Lanzavecchia, A., 2003. A role for Toll-like receptors in acquired immunity: up-regulation of TLR9 by BCR triggering in naïve B cells and constitutive expression in memory B cells. Blood 101, 4500- 4504.

Boonstra, A., Rajsbaum, R., Holman, M., Marques, R., Asselin-Paturel, C., Pereira, J.P., Bates, E.E., Akira. S., Vieira. P., Liu. Y.J., Trinchieri. G., O’Garra. A., 2006. Macrophages and myeloid dendritic cells, but not plasmacytoid dendritic cells, produce IL-10 in response to MyD88- and TRIF-dependent TLR signals, and TLR-independent signals. J. Immunol. 177, 7551- 7558.

Duddy, M., Niino, M., Adatia, F., Hebert, S., Freedman, M., Atkins, H., Kim, H.J., Bar-Or, A., 2007. Distinct effector cytokine profiles of memory and naïve human B cell subsets and implication in multiple sclerosis. J. Immunol. 178, 6092- 6099.

Fillatreau, S., Sweenie, C., McGeacht, M.J., Gray, D., Anderton, S.M., 2002. B cells regulate autoimmunity by provision of IL-10. Nature Immunol. 3, 944- 950.

Iwasaki, A., Medzhitov, R., 2004. Toll-like receptor control of the adaptive immune response. Nat. Immunol. 5, 987- 995.

Lampropoulou, V., Hoehlig, K., Roch, T., Neves, P., Gomez, E.C., Sweenie, C.H., Hao, Y., Freitas, A.A., Steinhoff, U., Anderton, S.M., Fillatreau, S., 2008. TLR-activated B cells suppress T cell-mediated autoimmunity. J. Immunol. 180, 1763- 1773.

Lehmann, D., Ben-Nun, A., 2002. Bacterial agents protect against autoimmune disease: I. Mice pre-exposed to Bordetella pertussis or Mycobacterium tuberculosis are highly refractory to induction of experimental autoimmune encephalomyelitis. J. Autoimmun. 5, 675- 690.

Marta, M., Andersson, A., Isaksson, M., Kampe, O., Lobell, A., 2008. Unexpected regulatory 
roles of TLR4 and TLR9 in experimental autoimmune encephalomyelitis. Eur. J. Immunol. 38, 565- 575.

McGeachy, M.J., Bak-Jansen, K.S., Chen, Y., Tato, C.M., Blumenschein, W., McClanahan, T., Cua, D.J., 2007. TGF- $\beta$ and IL-6 drive the production of IL-17 and IL-10 by T cells and restrain T(H)-17 cell-mediated pathology. Nat. Immunol. 8, 1390- 1397.

Poser, C.M., Paty, D.W., Scheinberg, L., McDonald, W.I., Davis, F.A., Ebers, G.C., Johnson, K. P., Sibley. W.A., Silberberg, D.H., Touetellotte, W.W., 1983. New diagnostic criteria for multiple sclerosis: guidelines for research protocols. Ann. Neurol. 13, 227- 231.

Prinz, M., Garbe, F., Schmidt, H., Mildner, A., Gutcher, I., Wolter, K., Piesche, M., Schroers, R., Weiss, E., Kirschning, C.J., Rochford, C.D., Bruck, W., Becher, B., 2006. Innate immunity mediated by TLR9 modulates pathogenicity in an animal model of multiple sclerosis. J. Clin. Invest. 203, 456- 464.

PRISMS (Prevention of Relapses and Disability by Interferon- $\beta$-1a Subcutaneously in Multiple Sclerosis) Study Group, 1998. Randomized double-blind placebo-controlled study of interferon $\beta$-1a in relapsing/remitting multiple sclerosis. Lancet 352, 1498- 1504.

Takeda, K., Kaisho, T., Akira, S., 2003. Toll-like receptors. Annu. Rev. Immunol. 21, 424- 432. The IFN- $\beta$ Multiple Sclerosis Study Group, 1993. Interferon beta-1b is effective in relapsing-remitting multiple sclerosis. Clinical results of a multicenter, randomized, double-blind, placebo-controlled trial. Neurology 43, 655- 661.

Vos, Q., Lees, A., Wu, Z.Q., Snapper, C.M., Mond, J.J., 2000. B-cell activation by T-cell-independent type 2 antigens as an integral part of the humoral immune response to pathogenic microorganisms. Immunol. Rev. 176, 154- 170.

Wolf, S.D., Dittel, B.N., Hardardottir, F., Janeway, C.A., 1996. Experimental autoimmune encephalomyelitis induction in B cell-deficient mice. J. Exp. Med. 184, 2271- 2278.

Zhang, X., Tang, Y., Sujkowska, D., Wang, D., Ramgolam, V., Sospedra, M., Adams, J., Martin. 
R., Pinilla. C., Markovic-Plese, S., 2008. Degenerate TCR recognition and dual DR2 restriction of autoreactive $\mathrm{T}$ cells: implications for the initiation of the autoimmune response in multiple sclerosis. Eur. J. Immunol. 38, 1297- 1309.

Zhang, X., Jin, J., Tang, Y., Speer, D., Sujkowska, D., Markovic-Plese, S., 2009. IFN $\beta-1 a$ inhibits the secretion of TH17-polarizing cytokines in human dendritic cells via TLR8 up-regulation. J. Immunol. 182, 3928- 3936. 


\section{Figure Legends}

Figure 1. TLR9 mediated decreased IL-10 but increased IL-12 (p40) production by B cells in MS.

The y-axis represents the mean IL-10, IL-12, and TNF- $\alpha$ levels in B cells stimulated with CpG DNA minus IL-10, IL-12, and TNF- $\alpha$ levels in unstimulated B cells ( $\Delta \mathrm{IL}-10, \Delta \mathrm{IL}-12$, and $\Delta$ TNF- $\alpha$ in pg/ml). (A) TLR9-mediated IL-10 production by B cells was significantly decreased in MS. Neither the disease phase nor DMT affected TLR9-mediated IL-10 production by B cells. (B) TLR9-mediated IL-12 (p40) production by B cells was significantly increased in patients with relapsing MS than in all other patients and controls. $(* p<0.05, * * p<0.01$ ) (C) No significant differences were found in TLR9-mediated TNF- $\alpha$ production by B cells among the groups.

\section{Figure 2. Surface-antigen profiles of B cells.}

(A) Example dot-plots of CD27, CD80, and CD86 expression on CD19-positive B cells in MS patients and healthy individuals. The numbers in the right upper quadrant represent the \% of positive cells in B cells. (B) No significant differences were found in the proportions of CD27-positive B cells (memory B cells) between MS patients and healthy individuals. The proportion of CD27-positive B cells was significantly higher in patients with relapsing MS than in MS patients treated with IFN $\beta$-1a or IFN $\beta-1 b$. (C) The proportion of CD80-positive B cells was significantly higher in patients with relapsing MS than in the other patient groups or controls. (D) The proportion of CD86-positive B cells was significantly lower in MS patients treated with IFN $\beta-1 b$ than in those not receiving DMT. $(* p<0.05)$

Figure 3. TLR9 MFI in B cells and CD27-positive B cells. 
(A) No significant differences were found in TLR9 MFI in B cells among all the study groups.

(B) However, TLR9 MFI in memory B cells was significantly lower in MS patients than in healthy individuals. (*p<0.01) (C) Representative results of TLR9 MFI in memory B cells. MFI values are indicated above the corresponding histograms. Shaded histograms indicate the staining pattern of isotype-control antibodies.

Figure 4. Correlation between TLR9-mediatedIL-10 production by B cells and TLR9 MFI in memory B cells

IL-10 production by B cells correlated positively with TLR9 MFI in memory B cells in all the subjects $(p<0.001, r=0.752)$. 
Table 1 Profiles of MS patients and healthy controls.

\begin{tabular}{|c|c|c|c|c|c|}
\hline & $\mathrm{N}$ & $\mathrm{F}: \mathrm{M}$ & $\begin{array}{c}\text { mean age } \\
\text { (years) }\end{array}$ & $\begin{array}{l}\text { disease } \\
\text { duration } \\
\text { (years) }\end{array}$ & EDSS \\
\hline \multicolumn{6}{|l|}{ MS } \\
\hline Relapsing phase & 6 & $4: 2$ & $32.3 \pm 14.8$ & $5.6 \pm 2.4$ & $4.3 \pm 2.9$ \\
\hline \multicolumn{6}{|l|}{ Remitting phase } \\
\hline Untreated & 10 & $8: 2$ & $37.7 \pm 12.7$ & $8.6 \pm 5.2$ & $2.1 \pm 1.5$ \\
\hline Treated with IFN $\beta-1 \mathrm{a}$ & 10 & $7: 3$ & $39.6 \pm 4.4$ & $9.2 \pm 5.5$ & $2.2 \pm 1.7$ \\
\hline Treated with IFN $\beta-1 b$ & 10 & $6: 4$ & $35.5 \pm 9.0$ & $8.1 \pm 5.2$ & $2.2 \pm 1.9$ \\
\hline Healthy control & 10 & $7: 3$ & $34.8 \pm 11.0$ & & \\
\hline
\end{tabular}

Data represent mean \pm SD. 
Figure 1

A

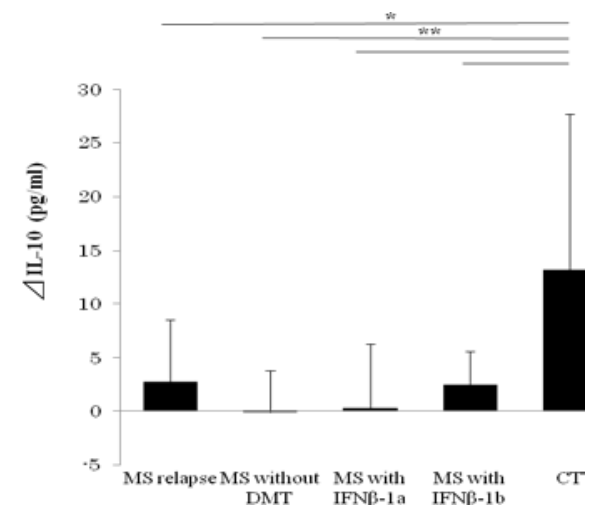

C

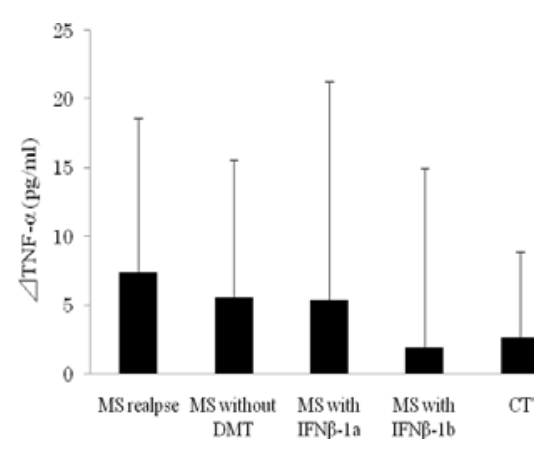

B

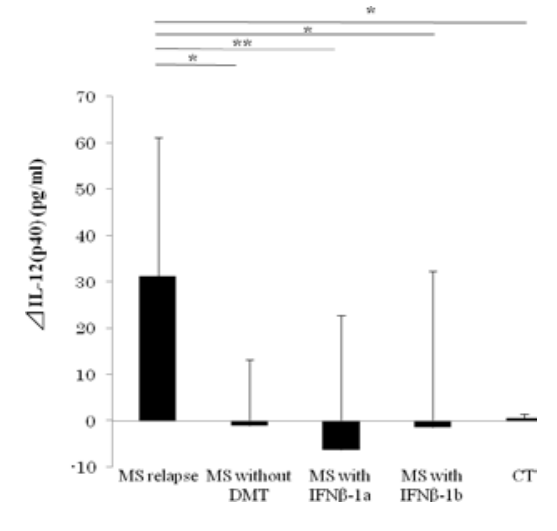


Figure 2

A
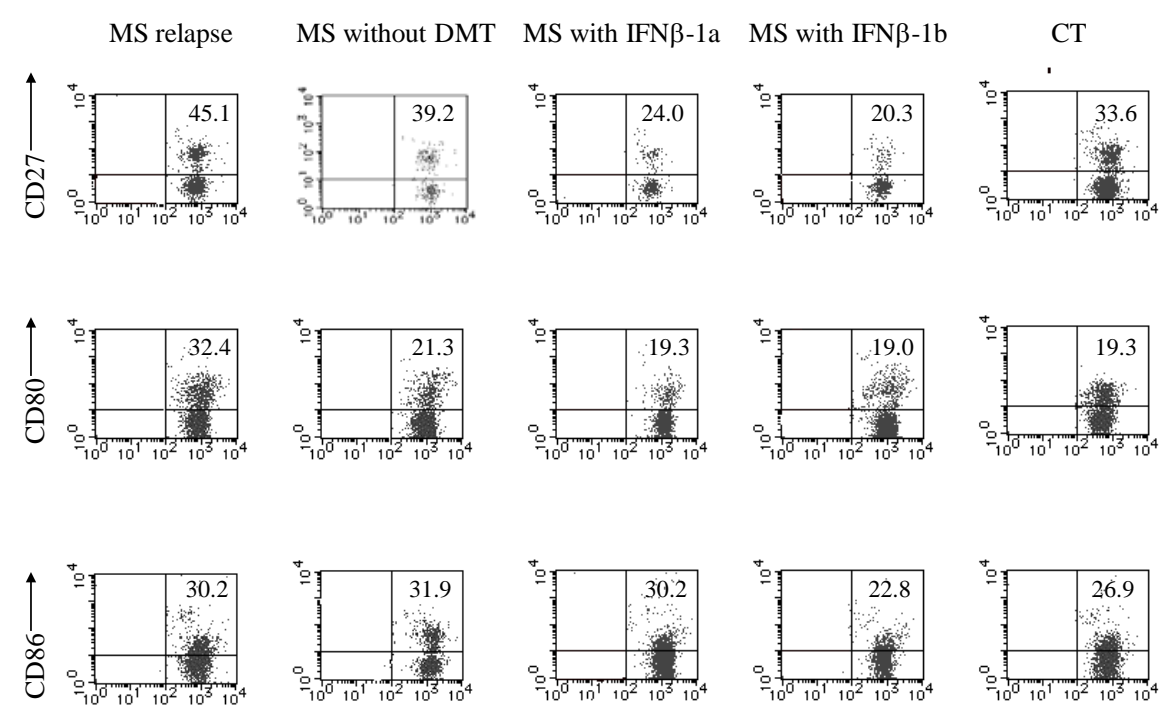

CD19

B

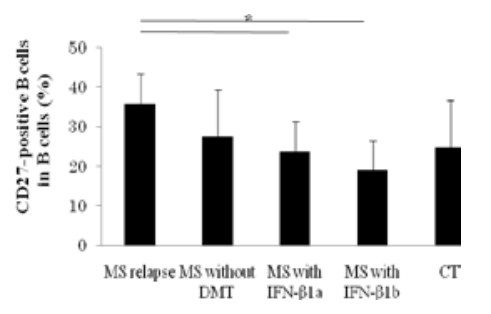

C

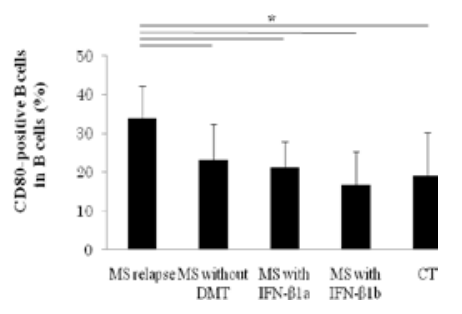

D

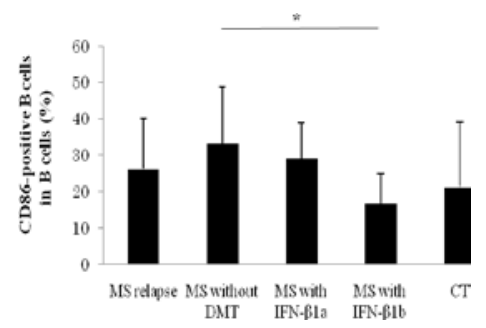




\section{Figure 3}

A

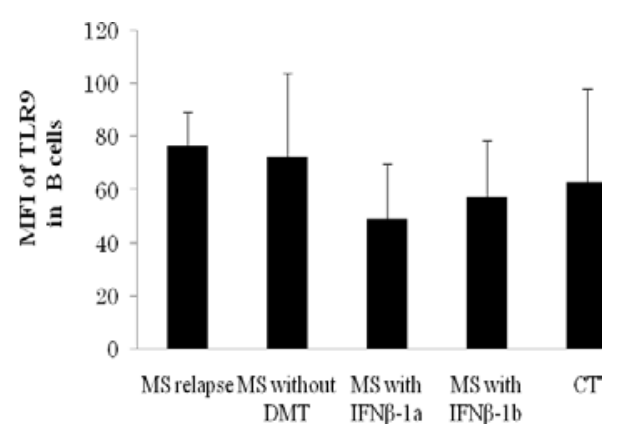

C

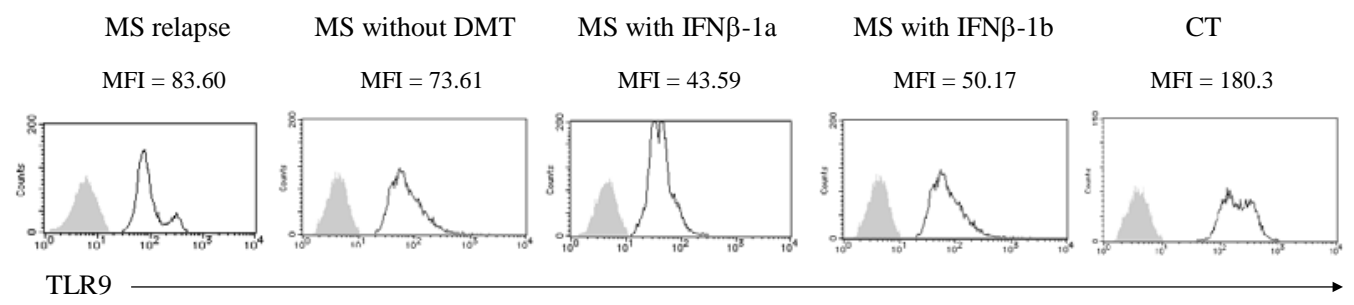

B

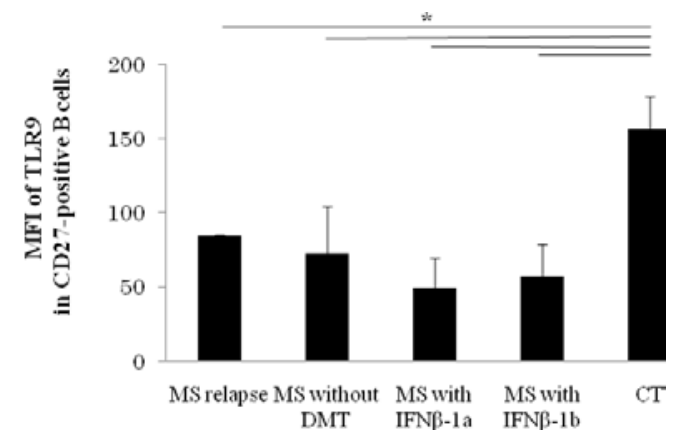


Figure 4

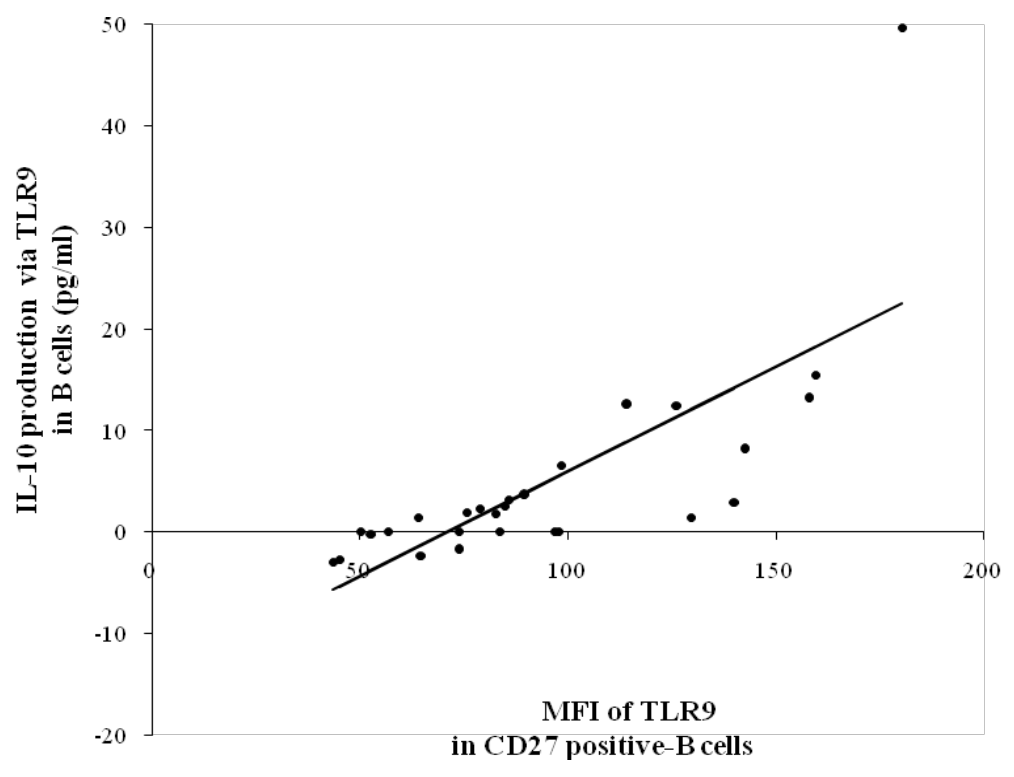

